\title{
EDITORIAL
}

\section{War on Staphylococcus aureus}

Journal of Perinatology (2014) 34, 803-804; doi:10.1038/jp.2014.129

Staphylococcus aureus has been a problem for both well and sick neonates for decades. As early as 1937, guidance for proper skin care of newborns was given to prevent staphylococcal infection. ${ }^{1}$ The first description of a penicillin-resistant $S$. aureus outbreak in the newborn nursery was in $1952,{ }^{2}$ when aureomycin, the first of the tetracyclines, was used for the treatment of pustular dermatitis, conjunctivitis and pneumonia. However, prevention of these outbreaks has proven to be very difficult. In the early 1960s, artificial colonization was employed, whereby infants were purposely colonized with less virulent $S$. aureus to prevent colonization with the more virulent strains. ${ }^{3}$ Constantly evolving, hospital-associated (HA) methicillin-resistant S. aureus (MRSA) emerged in the 1970s in adult intensive care units, and gradually infiltrated the neonatal intensive care unit (NICU). However, community-associated (CA) MRSA spread across the globe in the 1990s, causing necrotizing soft tissue and bone infections in people of all ages and affecting neonates colonized from mothers or nursery environment. Over the past several decades, infection control policies have been developed to combat S. aureus, including decolonization strategies, hand hygiene, surveillance and isolation procedures. However, despite many years of devising plans against $S$. aureus, the NICU remains a haven for this formidable pathogen.

Although more attention is paid to MRSA in both the lay and medical literature, during the last decade methicillin-susceptible S. aureus (MSSA) has become highly transmissible and infection is clinically indistinguishable from MRSA ${ }^{4,5}$ Reports in both adults and children hospitalized with CA-S. aureus infection show that epidemiologic factors do not distinguish CA-MRSA from CA-MSSA infections. ${ }^{6,7}$ Clinical presentations are also similar, with high prevalence of osteomyelitis, complications such as deep vein thrombosis and septic embolization to the lungs, and deep skin, soft tissue and lung infections. A clonal USA300 strain of S. aureus, which can be either MRSA or MSSA, is an important reason for this blurring. Only the presence of the staphylococcal cassette chromosomal mecA gene distinguishes MRSA. Although MSSA historically was considered endemic flora, MSSA infections can be as severe as MRSA infections in the pediatric population and may be more frequently associated with persistent bloodstream infection. Therefore, screening for both MRSA and MSSA is becoming increasingly important.

In this month's issue of the Journal, Popoola and Milstone ${ }^{8}$ attempt to show the existing data for the complicated issue of preventing $S$. aureus colonization and infections in the NICU. Unfortunately, few data are specific to the NICU population. It is difficult to determine which measures are the most effective for containment of MRSA because during an outbreak, many measures are instituted concurrently. In fact, it is likely that 'bundles' of containment and prevention techniques will be optimal, as in many infection control practices. A large, multicenter randomized controlled trial in adults in ICUs demonstrated that universal decolonization was effective at decreasing MRSA infections. ${ }^{9}$ However, we must be cautious at extrapolating these studies to neonates.

Mupirocin and chlorhexidine are not specific for S. aureus, and so colonization with other organisms is possible as the mucosal and skin surfaces re-populate after decolonization. Neonates are particularly vulnerable to perturbations in the establishment of their microbiota. The possibility of trading one problem for another is quite real. Mupirocin resistance is also possible. Bacteria are constantly changing to evade our antibiotics. This evolution is so rapid that bacteria consistently outpace the antibiotics that we create. The increase in multidrug-resistant organisms worldwide is increasing at such an alarming rate that public health officials are warning about a 'post-antibiotic era', when common infections are no longer treatable with our current arsenal of antibiotics. In addition to the threat of antibiotic resistance, nasal mupirocin alone will not work because both CA-MRSA and MSSA also colonize skin and perirectal areas as well as the nose, unlike old HA-MRSA. Finally, routine chlorhexidine bathing of neonates in the absence of an outbreak setting has been relatively contraindicated in young infants, because of the potential risk of neurotoxicity from hexachlorophene.

Despite the lack of NICU-specific data and the potential ramifications of decolonization, NICUs have adopted various decolonization protocols and infection control strategies because of the significant morbidity and mortality associated with S. aureus infections. Certainly, we could accept a particular level of risk associated with infection control strategies if these strategies prevented devastating $S$. aureus infections. However, recent data demonstrate a lack of efficacy of current aggressive decolonization protocols. Popoola and colleagues ${ }^{10}$ recently published their experience of implementation of intense infection control measures in their NICU over a 4-year period, including decolonization of infants and health-care workers, cohorting of colonized infants, routine surveillance cultures and increase in hand washing compliance. However, despite these aggressive measures, there was continued transmission of MRSA and subsequent infections occurred in colonized infants despite attempted decolonization.

Therefore, meticulous practice of infection prevention and control is our best weapon currently. With the blurry border between MRSA and MSSA, screening or isolating only for MRSA is short-sighted, particularly in neonates. Placing MRSA-positive infants on contact precautions, but not MSSA-colonized infants, is an out-dated and potentially dangerous approach to infection control in the NICU. Judicious use of antibiotics that alter flora, as well as removal of catheters that breech barriers as soon as possible are critical prevention tools.

The natural history of current $S$. aureus community-based epidemics suggests that control will be challenging and eradication impossible. However, it is our responsibility to keep the war going, as defeat is not an option. Optimal management and prevention of $S$. aureus infections in nurseries will require welldesigned, scientifically rigorous, prospective intervention and outcome studies. These are our opportunities to advance effective strategies, and to direct research and development of novel approaches that might include vaccination, specific antibody or antimicrobial prophylaxis, or topical decontaminants. However, no new strategy will replace the basic infection control practices of handwashing and maintaining vigilance to protect each patient one at a time.

\section{CONFLICT OF INTEREST}

The author declares no conflict of interest. 
AJ Carey ${ }^{1,2}$

${ }^{1}$ Division of Neonatology, Department of Pediatrics, Drexel University College of Medicine, St Christopher's Hospital for Children,

Philadelphia, PA, USA and

${ }^{2}$ Department of Microbiology and Immunology, Drexel University College of Medicine, Philadelphia, PA, USA

E-mail: alison.carey@drexelmed.edu

\section{REFERENCES}

1 Sanford HN. Care of the skin of the newborn infant. J Pediatr 1937; 11: 68.

2 Feldman F, Annunziata D. Staphylococcus aureus infections in the newborn infant. J Pediatr 1952; 41: 399-402.

3 Shinefield HR, Ribble JC, Boris M, Eichenwald HF. Bacterial interference: its effect on nursery-acquired infection with Staphylococcus aureus. I. Preliminary observations on artificial colonzation of newborns. Am J Dis Child 1963; 105: 646-654.

4 Kuint J, Barzilai A, Regev-Yochay G, Rubinstein E, Keller N, Maayan-Metzger A. Comparison of community-acquired methicillin-resistant Staphylococcus aureus bacteremia to other staphylococcal species in a neonatal intensive care unit. Eur J Pediatr 2007; 166: 319-325.
5 Carey AJ, Duchon J, Della-Latta P, Saiman L. The epidemiology of methicillinsusceptible and methicillin-resistant Staphylococcus aureus in a neonatal intensive care unit, 2000-2007. J Perinatol 2010; 30: 135-139.

6 Faden H, Rose R, Lesse A, Hollands C, Dryja D, Glick PL. Clinical and molecular characteristics of staphylococcal skin abscesses in children. J Pediatr 2007; 151: 700-703.

7 Miller LG, Perdreau-Remington F, Bayer AS, Diep B, Tan N, Bharadwa K et al. Clinical and epidemiologic characteristics cannot distinguish communityassociated methicillin-resistant Staphylococcus aureus infection from methicillinsusceptible S. aureus infection: a prospective investigation. Clin Infect Dis 2007; 44 471-482.

8 Popoola VO, Milstone AM. Decolonization to prevent Staphylococcus aureus transmission and infections in the neonatal intensive care unit. J Perinatol 2014 (this issue).

9 Huang SS, Septimus E, Kleinman K, Moody J, Hickok J, Avery TR et al. Targeted versus universal decolonization to prevent ICU infection. N Engl J Med 2013; 368: 2255-2265.

10 Popoola VO, Budd A, Wittig SM, Ross T, Aucott SW, Perl TM et al. Methicillinresistant Staphylococcus aureus transmission and infections in a neonatal intensive care unit despite active surveillance cultures and decolonization: challenges for infection prevention. Infect Control Hosp Epidemiol 2014; 35: 412-418. 